\title{
Composition and Interactions among Bacterial, Microeukaryotic, and T4-like Viral Assemblages in Lakes from Both Polar Zones
}

\begin{abstract}
Daniel Aguirre de Cárcer ${ }^{1}$, Carlos Pedrós-Alió ${ }^{2}$, David A. Pearce ${ }^{3,4,5}$ and Antonio Alcami ${ }^{1 *}$
${ }^{1}$ Centro de Biología Molecular Severo Ochoa, Consejo Superior de Investigaciones Científicas, Universidad Autónoma de Madrid, Madrid, Spain, ${ }^{2}$ CSIC, Institut de Ciències del Mar, Barcelona, Spain, ${ }^{3}$ British Antarctic Survey, Natural Environment Research Council, Cambridge, UK, ${ }^{4}$ Faculty of Health and Life Sciences, University of Northumbria, Newcastle Upon Tyne, UK, ${ }^{5}$ University Center in Svalbard, Lonyearbyen, Norway
\end{abstract}

In this study we assess global biogeography and correlation patterns among three components of microbial life: bacteria, microeukaryotes, and T4-like myoviruses. In addition to environmental and biogeographical considerations, we have focused our study on samples from high-latitude pristine lakes from both poles, since these simple island-like ecosystems represent ideal ecological models to probe the relationships among microbial components and with the environment. Bacterial assemblages were dominated by members of the same groups found to dominate freshwater ecosystems elsewhere, and microeukaryotic assemblages were dominated by photosynthetic microalgae. Despite inter-lake variations in community composition, the overall percentages of OTUs shared among sites was remarkable, indicating that many microeukaryotic, bacterial, and viral OTUs are globally-distributed. We observed an intriguing negative correlation between bacterial and microeukaryotic diversity values. Notably, our analyses show significant global correlations between bacterial and microeukaryotic community structures, and between the phylogenetic compositions of bacterial and T4-like virus assemblages. Overall, environmental filtering emerged as the main factor driving community structures.

Keywords: bacteria, microeukaryotes, T4 phages, pyrosequencing, diversity, lakes, polar

\section{INTRODUCTION}

The functioning of aquatic ecosystems is heavily dependent on the microbial food web (Azam et al., 1983), which consists of several components with different cellular (bacteria, archaea, and eukaryotes) or non-cellular (viruses) organizations. Polar freshwater environments embody some of the least human-impacted habitats on the planet (Convey and Stevens, 2007), and normally represent simple ecosystems with truncated food webs dominated by microorganisms. Arctic and Antarctic freshwater environments share common features, such as extreme annual cycles of temperature, sunlight, and ice phenology, and yet they are separated by long geographical distances. These characteristics make these environments a unique model to shed light on fundamental questions of aquatic microbial ecology, such as how communities vary across spatial scales and environmental gradients, or the association among different components of microbial life. 
Previous studies assessing the links between different components of microbial life have focused on local patterns, such as the microbial inventory of a single coral reef ecosystem (McCliment et al., 2012), or the time-series analysis of a specific marine site (Jones et al., 2013; Chow et al., 2014). All these studies reported co-occurrence patterns among members of the different components of microbial life. Another significant question in aquatic microbial ecology is the importance of dispersal limitations (Martiny et al., 2011). However, most studies tackling this issue have focused on marine samples, presumably showing enhanced connectivity related to global oceanic circulation (Sul et al., 2011; Ghiglione et al., 2012). On the other hand, the question of whether microbial species in freshwater bodies from both poles are the same or different is also of interest from the viewpoint of biogeography and genetic exchange (Bano and Hollibaugh, 2002).

The aim of this study is two-fold; first, to assess if similar microbial communities populate freshwater bodies from both polar zones. Second, to test whether the previously observed co-occurrence patterns among components of microbial life appear at a global scale, or instead represent local phenomena. To do so, we have studied the resident microbial communities from four Arctic lakes located in the Svalbard archipelago, and nine Antarctic lakes sampled across a latitudinal transect along the Antarctic Peninsula. Using a massive parallel sequencing approach targeting phylogenetic marker genes, we have studied the community structures of three components of microbial life: bacteria, microeukaryotes, and viruses. Since the latter lack a common phylogenetic marker gene, we have focused on the T4-like myovirus group. This group represents a diverse and abundant (Filée et al., 2005; Williamson et al., 2008) group of bacteria-infecting viruses (Wichels et al., 1998; Clokie et al., 2010) amenable to the proposed approach based on their g23 major capsid gene, which has been shown to serve as a phylogenetic proxy (Comeau and Krisch, 2008). After obtaining the community profiles, we carried out several analyses, both at the OTU level and based on overall community phylogenetic compositions, to study the relationships among the three components of microbial life studied, and with respect to biogeographical and limnological (both physical and chemical) factors.

\section{MATERIALS AND METHODS}

\section{Sampling and Community DNA Extraction}

Planktonic samples were taken from freshwater bodies in the Arctic (Spitsbergen Island, Svalbard archipelago, Norway), and along the Antarctic Peninsula (Table 1, Supplementary Figure 1). Lake Nordammen (SvL1) was completely frozen (samples represent a combination of existing melted top ice from three different sites), while Lake Tenndammen (SvL2) is a shallow lake with frozen surface at the time of sampling. All the other lakes had open waters at the time of sampling and representative samples of the water column were taken at different depths, except for Limnopolar lake where water was taken from $4 \mathrm{~m}$ depth. All the lakes were sampled around summer. A portable probe was deployed in situ before sampling the Antarctic sites to measure several limnological variables (Temperature, Conductivity, pH, Chlorophyll; Supplementary Table 1). Lake Green's measurement was deemed unreliable due to technical issues and hence data from this lake were removed from those analyses including limnological data.

Single ninety liter samples from each water body were filtered through a $30 \mu \mathrm{m}$ nylon mesh. Subsequent filtration by $0.45 \mu \mathrm{m}$ tangential flow filtration (TFF) using a Centramate holder (Pall) separated the free viral community (defined as the $<0.45 \mu \mathrm{m}$ fraction) from the cellular (and associated virus) fraction (defined as the fraction between 0.45 and $30 \mu \mathrm{m}$ ). Viral fractions were subsequently concentrated 100 times by 70 $\mathrm{kDa}$ TFF as described (López-Bueno et al., 2009; Aguirre de

TABLE 1 | Location, sampling date, and characteristics of the sampled environments.

\begin{tabular}{|c|c|c|c|c|}
\hline & Pole & Positiona & Date & Zoogenic input $^{b}$ \\
\hline Lake Domo* & Antarctic & $-62.64-60.97$ & 03/02/2010 & Very low \\
\hline Lake Refugio* & Antarctic & $-62.65-61.00$ & 03/02/2010 & Next to sea elephant colony \\
\hline Lake Limnopolar* & Antarctic & $-62.66-61.10$ & 01/02/2010 & Very low \\
\hline Caleta Cierva & Antarctic & $-64.16-61.01$ & 21/01/2010 & Nearby seabird colonies \\
\hline Green Island & Antarctic & $-65.31-64.15$ & 23/01/2010 & Accessible to seabirds \\
\hline Biscoe Point & Antarctic & $-65.43-65.48$ & 29/01/2011 & Very low \\
\hline Pourquoi-Pas Island & Antarctic & $-67.66-67.25$ & 27/01/2011 & Very low \\
\hline Avian Island & Antarctic & $-67.76-68.88$ & 25/01/2010 & Nearby seabird colonies \\
\hline Horseshoe Island & Antarctic & $-67.84-67.19$ & 25/01/2011 & Very low \\
\hline IR2 & Arctic & 78.0413 .69 & 27/09/2011 & Frequent seabird activity \\
\hline Lake Tunsijøen(IR1) & Arctic & 78.0513 .65 & 26/09/2011 & Frequent seabird activity \\
\hline Lake Nordammen(Svl1) & Arctic & 78.6316 .63 & 05/06/2012 & Accessible to seabirds \\
\hline Lake Tenndamen(Svl2) & Arctic & 78.1015 .03 & 06/06/2012 & Accessible to seabirds \\
\hline
\end{tabular}

a Lat Long.

$b_{\text {in }}$ situ observations.

* Lakes in Byers Peninsula, Livingston Island. 
Carcer et al., 2015). All samples were preserved at $-20^{\circ} \mathrm{C} /-80^{\circ} \mathrm{C}$ prior to DNA extraction. Cellular fraction community DNA was extracted using PowerSoil DNA isolation kit (MoBio, Carlsbad, CA) according to the manufacturer's instructions. Viral DNA was obtained from frozen stocks thawed at $4{ }^{\circ} \mathrm{C}$, and passed through a $25 \%$ sucrose cushion by centrifugation for $16 \mathrm{~h}$ at $60,000 \mathrm{~g}$ and $4{ }^{\circ} \mathrm{C}$. The pellets were re-suspended in $10 \mathrm{mM}$ Tris $\mathrm{pH} 8,1 \mathrm{mM}$ EDTA, and filtered using a $0.45 \mu \mathrm{m}$ syringe filter. Viral concentrates were then treated with DNAse I (500 U.ml ${ }^{-1}$ ), Nuclease S7 (420 U.ml $\left.{ }^{-1}\right)$, RNAse A $\left(100 \mu \mathrm{g} \cdot \mathrm{ml}^{-1}\right)$ and RNAse $\mathrm{H}$ (2 $\mathrm{U}$ per reaction) for $30 \mathrm{~min}$ at room temperature to remove free nucleic acids. Nuclease reactions were stopped with $12 \mathrm{mM}$ EDTA/2 mM EGTA, and viral capsids and envelopes were then disrupted with SDS $(0.5 \%)$ and proteinase $\mathrm{K}\left(200 \mu \mathrm{g} \cdot \mathrm{ml}^{-1}\right)$ treatment. Finally, viral DNA was extracted with phenolchloroform and ethanol precipitated.

\section{PCR Amplification of Community Marker Genes, and Massive Parallel Sequencing}

Bacterial 16S rRNA marker genes were amplified from the cellular fractions using primers $8 \mathrm{~F} 15 \mathrm{~B}\left(5^{\prime}-\right.$ [RocheAdapterB]AGAGTTTGATCCTGG-3') and 515R14AM (5'-[RocheAdapterA]-bc-TTACCGCGGCTGCT-3'; (Aguirre de Cárcer et al., 2011b)). All PCR reactions were carried out using $1 \mu \mathrm{l}$ of template DNA, $0.5 \mu \mathrm{l}$ Phusion High-fidelity polymerase (NEB), $20 \mathrm{nmol}$ dNTPs, 20 pmol of each primer, $1.5 \mu \mathrm{l} \mathrm{DMSO}, 0.4 \mathrm{mM} \mathrm{MgCl}_{2}$, in a final volume of $50 \mu \mathrm{l}$. Reaction conditions included an initial denaturation step of $30 \mathrm{~s}$ at $98^{\circ} \mathrm{C}$, followed by 25 cycles of $10 \mathrm{~s}$ at $98^{\circ} \mathrm{C}, 30 \mathrm{~s}$ at $53^{\circ} \mathrm{C}, 30 \mathrm{~s}$ at $72^{\circ} \mathrm{C}$, and a final elongation step of $5 \mathrm{~min}$ at $72^{\circ} \mathrm{C}$. For the analysis of the microeukaryotic and T4-like viral assemblages we followed a validated two-step barcoding strategy (Aguirre de Cárcer et al., 2011b) that allows a universal set of bar-coded sequencing primers to be appended to an amplified PCR product without introducing discernible biases. In the first step, one of the target-specific primers is modified to include a linker sequence. After amplification, a second primer consisting of the bar code and linker is used to tag the amplicon. The eukaryotic community in the cellular fractions was analyzed using primer sequences targeting the 18S rRNA gene (Bates et al., 2013; F515; 5'-GTGCCAGCMGCCGCGGTAA$3^{\prime}$ and R1119 5'-GGTGCCCTTCCGTCA-3'). The T4-like virus communities in both cellular and free viral fractions were assessed using primer sequences (Filée et al., 2005) targeting the g23 major capsid protein gene (MZIA1bis $5^{\prime}$-GATATTTGIGGIGTTCAGCCIATGA-3' and MZIA6 5'CGCGGTTGATTTCCAGCATGATTTC- $3^{\prime}$ ). In this case, we performed an initial amplification using the unmodified (no linker or 454-adaptor sequences) primers (45 cycles, melting temperature of $50^{\circ} \mathrm{C}$ ), followed by agarose gel extraction of DNA bands of appropriate size. The resulting products were re-amplified using the modified primers (10 cycles), at this point linking with the two-step protocol. The primer pairs employed in this study have been reported to target most known sequences within their target groups. Final concentrations of PCR products were measured using a PicoGreen dsDNA Assay
Kit (Life Tech.), equal amounts for each sample pooled, agarose gel-extracted using the QIAquick Gel Extraction Kit (QIAGEN), and sequenced using a Roche 454 FLX sequencer with titanium chemistry. Bacterial and Eukaryotic profiles from each lake were generated in triplicate (hence three different barcodes/reactions per sample) to mitigate potential reaction-level PCR biases (Bates et al., 2013). All sequences have been deposited at ENA under id PRJEB10639.

\section{Sequence Processing and Data Analysis}

The sequences obtained were denoised with ACACIA (Bragg et al., 2012) then processed using QIIME (Caporaso et al., 2010). Sequences were first assigned to each sample using their respective barcodes. They were next filtered for correct length and quality values (Maximum number of ambiguous bases; 5. Mean quality score; $>25$. Maximum homopolymer length; 6. Maximum mismatches in primer; 0. Chimera removal with usearch). Later, all sequences were grouped into operational taxonomic units (OTUs) at 0.97 distance thresholds using the Uclust algorithm, and OTUs not appearing in at least two replicates across each dataset were discarded to eliminate noise and possible artifacts. At this point, the results from the persample technical triplicates were pooled to obtain per-sample community profiles. The resulting sample-by-OTUs matrices were subsampled to the minimum number of sequences at any given site (independently for each marker gene dataset) to normalize sampling efforts, and singletons were removed (Aguirre de Cárcer et al., 2011a). Additionally, representative sequences from each bacterial and eukaryotic OTU (the most abundant sequence of that OTU across the dataset) were confronted against the Greengenes (DeSantis et al., 2006)/Silva (Quast et al., 2013) reference alignments (for 16S and 18S sequences respectively) for taxonomic affiliation. Shannon diversity indices, Chaol richness estimates, and percentage of shared OTUs between samples were obtained using dedicated QIIME scripts.

Statistical and analytical procedures were carried out in $R(\mathrm{R}$ Core Team, 2015).

Overall differences between poles or sampling fractions (only for T4-like viruses) in terms of richness, diversity, percentage of shared OTUs, and relative abundance of major eukaryotic taxa were assessed using $t$-tests (t.test function).

The relationships between diversity values and latitude, limnological variables, or the relative abundance of major taxonomic groups were explored through linear regression. Bootstrapping was conducted to test whether or not the slope of the regression was significantly different from zero (boot function within package of the same name, Davison and Hinkley, 1997), and alternatively, performing a Wald test for multiple coefficients (f.robftest function from package sfsmisc).

The existence of correlation between percentages of shared OTUs and geographical distance or limnological profiles was assessed through Mantel tests (mantel.randtest, package ade4).

The initial exploration of community structure data was carried-out through double principal coordinates analysis (DPCoA) using functions from the ade4 package. This is an ordination method that takes into account phylogenetic 
(genetic distance) relatedness between OTUs when explaining variation in the data, hence quantifying community dissimilarity based on phylogenetic relatedness. Statistical significance of $a$ priori community groupings (e.g., pole of origin) was tested by between class analysis (BCA) and constrained double principal coordinates analysis (cDPCoA) (phylogenetic-aware) (Dray et al., 2015) available in ade4. Identification of limnological variables correlated with community structure was undertaken by analysis with instrumental variables (IV) (Baty et al., 2006) using the pcaiv function of the same package. Finally, associations among community structures derived from different marker genes, as well as with existing environmental data (limnological profiles, geographic distance, latitude) were assessed with Mantel tests using Bray-Curtis and Rao (phylogenetic-aware) distance matrices derived from the community profiles.

\section{RESULTS}

\section{OTUs Richness and Diversity}

Samples from four water bodies in Spitsbergen Island $\left(78^{\circ} \mathrm{N}\right.$, Svalbard, Norway) and nine water bodies along the Antarctic Peninsula $\left(62^{\circ}-67^{\circ} \mathrm{S}\right)$ were collected in three different years (Table 1, Supplementary Figure 1). From these samples, community DNA was prepared using both cellular and free virus fractions, and bacterial 16S rRNA, eukaryotic 18S rRNA and T4like virus g23 major capsid protein genes were PCR-amplified and sequenced using Roche 454 technology. The strategy employed produced 78000 (16S), 23000 (18S), and 38000 (g23) sequences passing the initial quality control. Subsequent filtering resulted in $5302 \pm 1040$ (16S), $1805 \pm 440$ (18S), and $1514 \pm 496$ (g23) sequences per sample (Supplementary Table 1). However, we were unable to produce reliable g23 sequence data for a small subset of the sites and fractions (4 out of 26), and hence these data points were removed from some analyses. Clustering of sequences at $97 \%$ similarity produced 1548 (16S), 704 (18S), and 176 (g23) OTUs in total. The number of bacterial OTUs per sample ranged between 126 in Horseshoe Lake and 864 in Lake SvL1 (Supplementary Table 1). The number of singletons was moderate in all cases, resulting in Chao 1 estimates that were only about twice as large as the retrieved OTUs (ranging between 121 and 1466). Corresponding values were lower for eukaryotes with values ranging between 87 OTUs in Lakes Biscoe and SvL2 and 418 in Lake IR2 (average 197), and even lower for viruses. Interestingly, viral richness was higher in the cellassociated (average 33 OTUs) than in the free viral fractions (average 21 OTUs, Supplementary Table 1) although difference did not reach statistical significance $(p>0.05)$.

The microbial communities studied shared a noticeable percentage of OTUs: averaging 14, 10.2 and 5.8\% for bacteria, eukarya and viruses respectively (Table 2). Communities from the same polar zone shared a significantly higher percentage of OTUs. The only exception was the Arctic bacterial assemblages, which only shared approximately $10 \%$ of their OTUs among themselves. In the case of T4-like viral assemblages, a higher percentage of shared OTUs between the free viral and cellular fractions arising from the same sample was observed when compared to the inter-lake average $(31.4 \%$ vs.
TABLE 2 | Shared OTUs.

\begin{tabular}{lllr}
\hline & \multicolumn{1}{c}{$\mathbf{1 6 S}$} & $\mathbf{1 8 S}$ & $\mathbf{9 2 3}$ \\
\hline Overall & $14.0 \pm 4$ & $10.2 \pm 2.4$ & $5.8 \pm 11.6$ \\
Intra-Antarctic & $19.4 \pm 4^{* * \star}$ & $13.7 \pm 2.2^{\star * *}$ & $5.7 \pm 2.2^{\star}$ \\
Intra-Arctic & $10.1 \pm 1.1$ & $11.7 \pm 2.8^{*}$ & $10.9 \pm 9.3^{*}$ \\
\hline
\end{tabular}

Numbers represent averaged values (\%) of geometric means of the pairwise comparisons. ${ }^{\star} p<0.05 ;{ }^{* \star *} p<0.0005$. Paired $t$-tests. Comparison with respect to the overall shared OTUs.

overall 5.8\%, $p<0.0005$, respectively). For the Antarctic bacterial and microeukaryotic data sets, we found no significant correlation between the percentage of shared OTUs and either geographical distance or limnological profiles based on temperature, conductivity, $\mathrm{pH}$, and Chlorophyll (Supplementary Table 2).

Shannon's entropy index was used to study community diversity (Table 3). No significant differences were found when comparing diversity values between the two polar zones, either for the bacterial (Antarctic; $4.7 \pm 1.1$, Arctic; $4.7 \pm 0.8$ ) or microeukaryotic assemblages (Antarctic; $3.9 \pm 1.1$, Arctic; $4.0 \pm 1.4)$. Also, for the Antarctic datasets, no correlation was observed between community diversity values and latitude along the studied transect. Moreover, observed diversity values and recorded limnological variables (Supplementary Table 2) only correlated in the case of 16S-based bacterial community diversity and Chlorophyll $a$ content, where a positive correlation was found (Slope $0.85, R^{2}=0.3$ ), although the observed correlation only reached significance $(p<0.05)$ with one of the two statistical tests applied (Figure 1A). Strikingly, a negative correlation (Slope $-0.54, R^{2}=0.21$ ) was observed between bacterial and microeukaryotic diversity values (Figure 1B), with the negative association reaching a noticeable degree of significance with both statistical tests applied (linear regression bootstrapping $p<0.05$; Wald test $p=0.078$ ). Subsequent analyses failed to show significant correlations between diversity indices of bacterial and microeukaryotic assemblages and the relative abundances of any major taxonomic groups.

In the case of the T4-like viral assemblages (Table 3) no significant differences in diversity estimates were observed with regards to pole of origin. $16 \mathrm{~S}$ and $18 \mathrm{~S}$-based values obtained for the same samples gave no significant correlations when compared to T4-like virus values. Moreover, geographical (distance, latitude) and limnological parameters available for the Antarctic sites did not show an influence on T4-like virus community diversity. Lastly, the cellular fractions were more diverse than their free viral fraction counterparts, yet such differences did not reach statistical significance $(p=0.11)$. One peculiar case was that of the free viral fraction of Lake Biscoe that consisted of a single OTU. This OTU was also detected at $11 \%$ relative abundance in the cellular fraction of Lake SvL1 in the Arctic suggesting that it was not an artifact.

\section{Taxonomic Compositions}

Bacterial community profiles (Figure 2A) were dominated by sequences classified as order Burkholderiales (39\%), containing 
mainly representatives of families Oxalobacteriaceae (13\%) and Comamonadaceae (22\%). Bacteroidetes-related sequences were also very abundant (35\%), dominated by Flavobacteriaceae (29\%), and Sphingobacteriales (5\%). Actinobacteria-related sequences were abundant (8\%), mainly corresponding to the Microbacteriaceae (3\%), and ACK-M1 (4\%) clades. Finally, Cyanobacteria-related sequences averaged $8 \%$, yet a closer inspection revealed that, although a few sequences belonged to the Synechococcophycidae and Nostocaceae clades, the great majority (94\%) corresponded to algal chloroplast-related sequences. When the taxonomic composition was examined separately for each lake, substantial differences emerged (Figure 3A). The predominance of Betaproteobacteria and Bacteroidetes was apparent, but some lakes were dominated by the former, such as Cierva, Horsheshoe, SvL2, and Svl1, while others were dominated by Bacteroidetes, like Domo, Pourquois-Pas, and IR2. Chloroplasts appeared only in lowlatitude Antarctic lakes. Finally, Actinobacteria were abundant in several Antarctic lakes but only in one Arctic lake. Interestingly, within the Betaproteobacteria, family Oxaloacetaceae was the most abundant in two Arctic lakes (SvL1 and Svl2), while

TABLE 3 | Shannon's diversity indices.

\begin{tabular}{lcccc}
\hline & Bacteria & Eukarya & Virus $^{\mathbf{f}}$ & Virus $^{\mathbf{c}}$ \\
\hline Domo & 4.28 & 2.95 & $\mathrm{NA}$ & 2.25 \\
Refugio & 6.50 & 4.46 & $\mathrm{NA}$ & $\mathrm{NA}$ \\
Limnopolar & 4.95 & 3.99 & 3.01 & 2.84 \\
Cierva & 3.37 & 5.61 & $\mathrm{NA}$ & 1.52 \\
Green & 6.45 & 2.31 & 2.63 & 2.39 \\
Biscoe & 4.72 & 2.68 & 0 & 2.16 \\
Pourquoi-pas & 4.10 & 4.76 & 0.39 & 1.94 \\
Avian & 4.57 & 5.04 & 2.28 & 2.29 \\
Horseshoe & 3.51 & 4.12 & 1.36 & 1.84 \\
IR2 & 4.00 & 5.67 & 1.95 & 1.34 \\
Tunsijøen IR1 & 5.29 & 4.80 & 1.32 & 1.45 \\
Tenndamen SvL2 & 5.62 & 2.26 & 1.83 & 1.99 \\
Nordammen SvL1 & 4.08 & 3.58 & 1.84 & 4.17 \\
\hline
\end{tabular}

${ }^{f}$ Free virus fraction.

${ }^{c}$ Cellular fraction.
Comamonadacea was the most abundant family in most other lakes.

Globally, microeukaryotic assemblages (Figure 2B) were dominated by sequences classified as Stramenopiles (26\%), Viridiplantae (19\%), and Alveolata (15\%). A major difference between the two polar zones became evident when the composition was examined for the different lakes separately (Figure 3B): Arctic lakes were clearly dominated by Alveolata while the Antarctic lakes had mostly Viridiplantae and Stramenopiles, with differences reaching statistical significance $(p<0.05)$. The Stramenopiles belonged mostly to the Chrysophyceae (12\%) and Ochromonadaceae (6\%). The Viridiplantae were mostly Chlorophyta, and the Alveolata mostly Dinophyceae (8\%). Some Antarctic lakes were dominated by Viridiplantae while others were dominated by Stramenopiles. Other groups were present in some lakes only, and in smaller proportions. Cercozoa were one such group. They were abundant in two Arctic and one Antarctic lake and present in a few more lakes. There were also sequences related to fungi. In particular, one extremely abundant (52.4\%) OTU in Lake SvL2 related to the Basidiomycota, and Chytridiomycota-affiliated sequences in lake IR2 (12.5\%). Another interesting case was that of a Telonema OTU that was very abundant in Lake IR1. Telonema is a widely distributed marine heterotrophic flagellate that forms a deep branch in the tree of eukaryal life. Finally, an average 13\% of eukaryotic sequences could not be properly assigned, and 17\% were related to uncultured eukaryotes.

Overall, bacterial community compositions for the studied environments were dominated by members of the Burkholderiales, Bacteroidetes, and Actinobacteria, the same groups found to dominate both Polar and non-Polar freshwater ecosystems (Newton et al., 2011; Logares et al., 2013; Barberán and Casamayor, 2014; Vick-Majors et al., 2014). The same was true for the microeukaryotic assemblages, where the dominance of Stramenopiles, Chlorophyta, and Alveolata, is in line with previous knowledge that most eukaryotes in other Antarctic lakes were photosynthetic microalgae (Wilkins et al., 2013; VickMajors et al., 2014). Phototrophic microeukaryotes accounted for about $60 \%$ of the eukaryotic sequences. On the other hand, $16 \mathrm{~S}$ sequences provided very few sequences of free-living cyanobacteria and a substantial amount of chloroplast sequences. This indicates that the primary producers in the studied pelagic
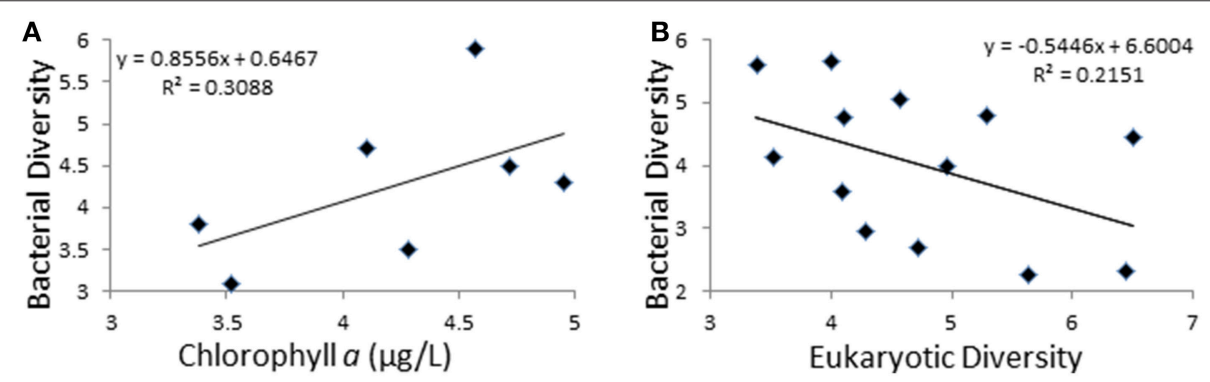

FIGURE 1 | Exploration of diversity trends. (A) Relationship between bacterial diversity (Y-axis) and chlorophyll a concentration (X-axis). (B) Relationship between bacterial (Y-axis) and microeukaryotic diversity (X-axis). Diversity is represented by Shannon's indexes and Chlorophyll a is in $\mu \mathrm{g} / \mathrm{L}$. 

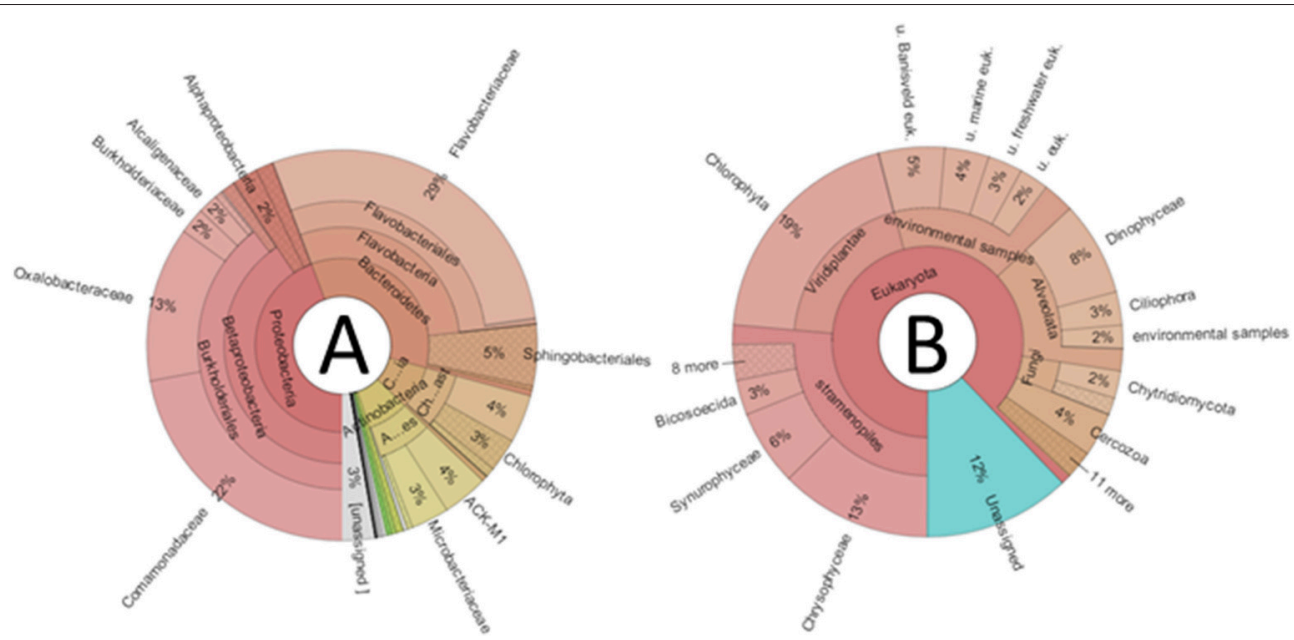

FIGURE 2 | Krona graphs depicting averaged relative abundances of taxonomic groups in the studied communities. (A), Bacteria; (B), Eukarya; u, uncultured; euk, eukaryote.

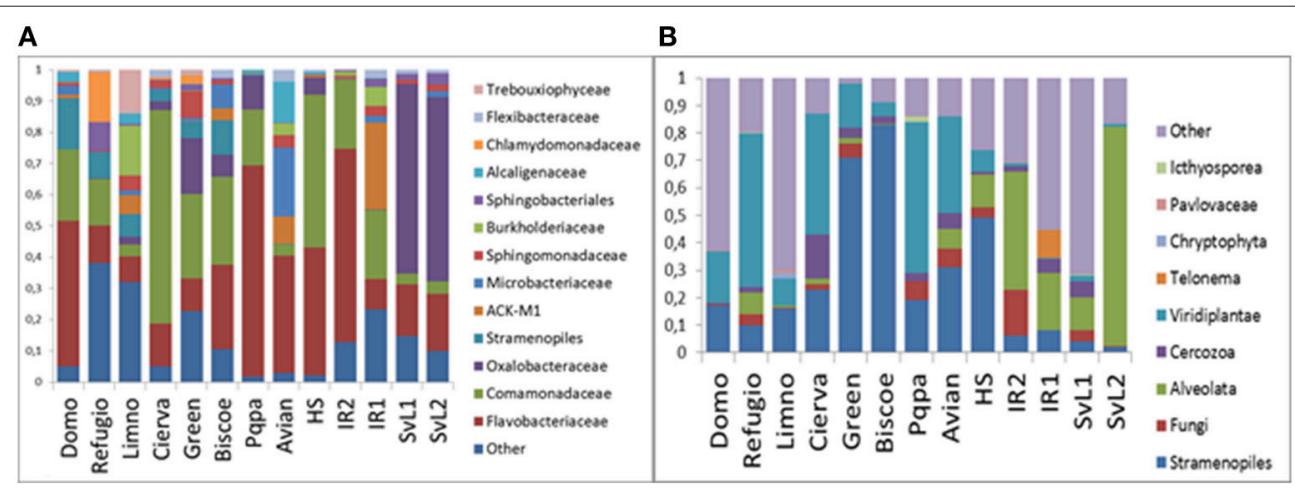

FIGURE 3 | Per-lake relative abundances of taxonomic groups in the studies communities. (A) Bacteria. (B) Eukarya.

ecosystems were eukaryotes, which is in contrast to the dominant role of Cyanobacteria in the benthos of Antarctic freshwater systems (Wharton et al., 1983; Vincent, 2000). However, our results are in agreement with the fact that viral metagenomes from polar freshwater environments were found to be dominated by likely microeukaryote-infecting viruses (López-Bueno et al., 2009; Aguirre de Carcer et al., 2015), and with results from microscopy observations (Izaguirre et al., 1998).

\section{Community Structure}

Double principal coordinates analyses were used to explore the relationships between phylogenetic community compositions at each site. The major source of variation in the bacterial data set (Figure 4A) related to the relative proportions of sequences affiliated as Flavobacteria and Betaproteobacteria. Lakes Avian, Domo, IR2, and Pourquois-Pas showed increased abundances of Flavobacteria and reduced abundances of Betaproteobacteria, whereas Cierva, SvL1, SvL2, and Green exhibited the opposite pattern. On the other hand, variation in the microeukaryotic data set was principally related to the relative proportions of sequences affiliated to the Stramenopiles, Alveolata, and Viridiplantae clades (Figure 4B). As already mentioned Antarctic communities had significantly $(p<0.05)$ lower relative abundances of Alveolata-affiliated sequences and higher proportions of those affiliated to the Stramenopiles and Viridiplantae clades, when compared to the studied Arctic sites. Furthermore, the results confirmed two opposing clusters of Antarctic sites along a Stramenopiles-Viridiplantae axis.

For the remaining exploration of the datasets, we carriedout two types of analyses (Table 4): those based solely on OTUs relative abundances (Mantel tests of Bray-Curtis distances, Between Class Analysis), and analyses based on both OTUs relative abundances and phylogenetic relatedness between OTUs (Mantel tests of Rao distances, constrained Double Principal Coordinates Analysis). First, we examined the relationship between the composition of the cellular components and both geographical distance and limnological parameters (Table 4A). We found no significant association between either component and geographic distance or latitude. On the other hand, we observed a significant correlation between 


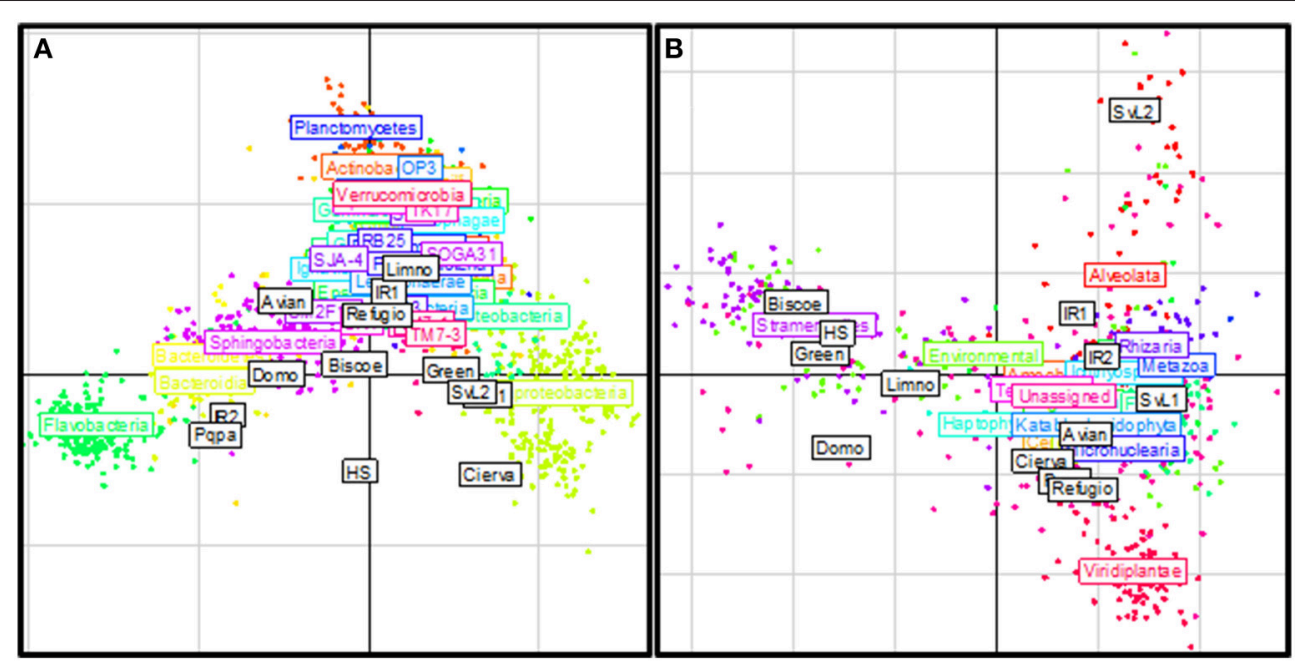

FIGURE 4 | Phylogenetic composition similarities between polar freshwater assemblages. The figures depict results from Double Principal Coordinates Analyses (DPCOA) based on 16S (A) and 18S (B) marker gene profiles. The analysis ordinates the sites (white boxes) based on the phylogenetic distances and per-site relative abundances of OTUs. The position of each point (OTU) represents its association with each site. Points are colored and collectively labeled based on their taxonomic affiliation.

TABLE 4 | Community structure analyses.

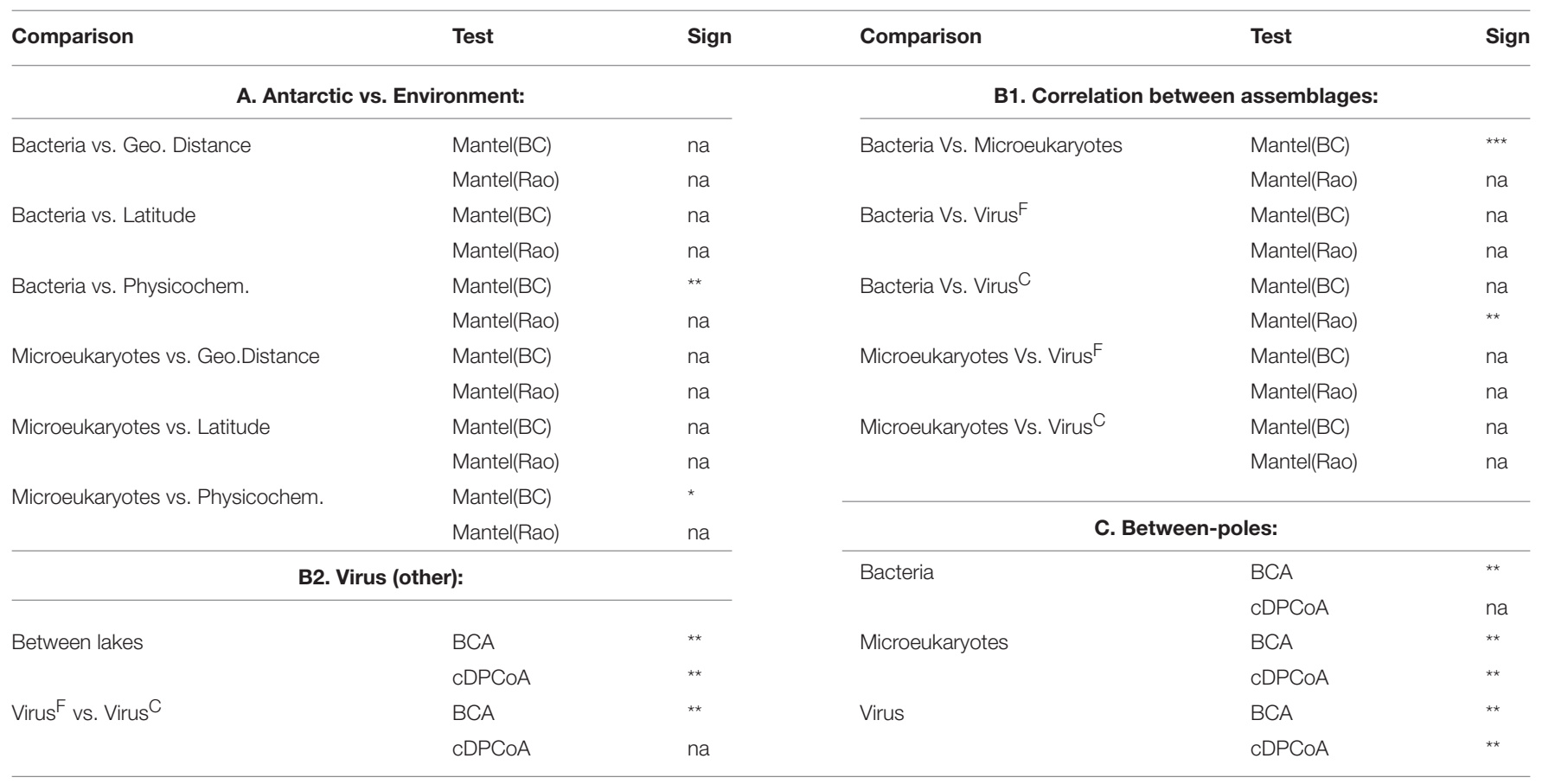

Tests: Mantel(BC), [Mantel test based on Bray-Curtis distances]; Mantel(Rao), [Mantel test based on Rao distances\{Phylogenetic]]; BCA, [Between class analysis]; cDPCoA, [Constrained Double Principal Coordinates Analysis \{Phylogenetic\}. Sign: na $(p>0.1),{ }^{\star \star \star}(p<0.001),{ }^{* *}(p<0.05),{ }^{\star}(p<0.1) .{ }^{F}$ Free virus fraction, ${ }^{C}$ Cellular fraction.

bacterial community structure and the limnological parameters recorded in the Antarctic sites, and marginal association $(p=$ 0.068 ) between the same parameters and the microeukaryotic assemblages. In both cases, associations seemed to be related to temperature and conductivity (analysis with instrumental variables based on Bray-Curtis distances; $p<0.05$ ). Nevertheless, comparisons became statistically not significant when taking into account the overall phylogenetic composition of the communities.

Next, we analyzed the correlations among the different microbial components (Table 4B). Bacteria and microeukaryotic assemblages showed a significant correlation employing Bray-Curtis distances, yet statistical significance disappeared when using Rao distances (phylogenetic-aware). On the 
contrary, bacterial and viral cellular fractions showed a significant correlation with Rao distances but not with BrayCurtis distances. As expected, cellular and free virus fraction T4-like virus assemblages from the same lake were more similar to each other than to those of the other lakes, also when accounting for the genetic similarities among sequences. Interestingly, we observed a significant discrimination between virus assemblages arising from either cellular or free viral fractions with Bray-Curtis distances, although such differences disappeared when Rao distances were considered.

Finally, we examined potential differences between the two polar zones (Table 4C). Bacterial assemblages from the same pole were more similar to each other than to those of the other pole, yet the overall genetic diversity of both poles was undistinguishable. In the cases of the microeukaryotic and T4like virus assemblages, on the other hand, both poles were shown to harbor distinct community structures, also when accounting for the genetic diversity of the OTUs.

\section{DISCUSSION}

\section{Composition of Polar Freshwater Microbial Communities}

The phylogenetic analysis clustered the lakes on the basis of their microeukaryotic composition, with Arctic lakes bearing higher proportions of Alveolata/Dinophyceae, and Antarctic lakes partitioned along a Viridiplantae (Avian, Cierva, Pourquois-Pas, and Refugio) vs. Stramenopiles (Limnopolar, Biscoe, Horseshoe, Green, and Domo) axis. This second axis roughly matches the classification of the lakes according to their higher or lower Chlorophyll concentration (Supplementary Table 2). Interestingly, this partitioning is also in line with that reported by Izaguirre et al. (1998) for lakes in Hope Bay (Antarctica), where trophic status was shown to impact microeukaryotic assemblages: more oligotrophic lakes were dominated by Chrysophyceae (Stramenopiles), and more eutrophic lakes were dominated by Chlorophyceae (Viridiplantae). These authors reported that trophic status of the freshwater bodies in Hope Bay depended strongly on their proximity to bird colonies. In the present study most lakes did not appear to have a strong influence of marine birds and mammals. However, partitioning along the abovementioned axis correlated with estimated zoogenic input (Table 1). Moreover, Lake Refugio was surrounded by substantial numbers of elephant seals, likely explaining the very high value of Chlorophyll in this lake and the dominance of Viridiplantae.

We detected significant differences between T4-like virus assemblages arising from the cellular and free virus fractions. However, such differences appeared only at the level of OTUs, and not when accounting for the overall phylogenetic compositions of the fractions. These observations may indicate that different phylogenetically-related viral OTUs have a preference to localize in one or the other fraction. This fact may have important methodological consequences, since most protocols used to study overall viral assemblages (e.g., through viral shot-gun metagenomics) rely on the study of the free viral fraction exclusively, in order to reduce the massive proportion of undesired bacterial DNA in the resulting datasets (López-Bueno et al., 2009; Fancello et al., 2013; Brum and Sullivan, 2015). Also, the free virus fraction was less diverse than the cellular fraction (e.g., intra-cellular and membrane-attached viruses). Moreover, a significant correlation was found between bacterial and T4like viral assemblages present in the cellular fractions. Altogether, these results indicate that studying solely free viral fractions may result in a rather biased picture of the ecology of viruses.

\section{Limnological Parameters Drive Antarctic Community Structures}

We could not find evidence for latitude or geographical distance having a significant influence on the community composition of Antarctic sites (Table 4A). This was true for both the bacterial and the microeukariotic components. On the other hand, limnological parameters correlated with both bacterial and microeukaryotic community structure (Table 4A). The same weak or inexistent short to mid-range biogeographical patterns in microbial communities, combined with strong environmental filtering have previously been observed in similar ecosystems, such as Antarctic lakes (Logares et al., 2013), or sub-arctic and Arctic marine environments (Winter et al., 2013). Subsequent analyses revealed that both conductivity and temperature co-varied with community structure. However, it is important to note that in the present dataset temperature and conductivity were correlated (Supplementary Table 1). The influence of temperature was not unexpected, since it had previously been shown to affect bacterial community structure in northern European lakes (Lindström et al., 2005). The effects of temperature and conductivity were significant exclusively on the Bray-Curtis distances, but they did not affect overall phylogenetic compositions (Table 4A). The fact that the latter remained unaffected by environmental variables likely indicates the existence of ecologically-redundant, phylogenetically-related, OTUs fit to slightly different limnological scenarios. We had anticipated significant $\mathrm{pH}$ effects, as they had previously been reported in studies of high altitude (Barberán and Casamayor, 2014; $\mathrm{pH}$ range 4.5-9), northern Europe (Lindström et al., 2005; pH 5.5-8.7), and Wisconsin lakes (Yannarell and Triplett, 2005; $\mathrm{pH}$ 5.4-8.6). It is possible that we did not detect $\mathrm{pH}$-related effects due to the reduced dataset size. On the other hand, $\mathrm{pH}$ range along our sites did not reach two units (5.8-7.7), while in all abovementioned studies the range exceeded 3 units.

\section{Bipolar Patterns in Community Structures}

The overall percentages of shared OTUs were considerable (between 6 and 19\%), further substantiating the idea that while each lake has a particular community structure, many lakes can harbor the same OTUs (Newton et al., 2011). Nevertheless, the percentages of shared bacterial OTUs between lakes from different poles were noticeably lower than those observed between marine Arctic and Antarctic bacterial assemblages ( $c a$. 20-30\%) (Ghiglione et al., 2012), suggesting a stronger effect of water-borne bacterial dispersal and (or) a greater variability in the physicochemical environment between lakes vs. ocean samples. In particular, Ghiglione et al. (2012) found indications that deep water circulation might be responsible for dispersal of OTUs 
from one polar ocean to the other. Lakes are not communicated by oceanic circulation and, thus, dispersal can only occur through the air or through migrating Arctic terns. In this regard, it is not surprising that the number of shared OTUs is lower in freshwater bodies than in marine polar areas. Additional examples of similar or even identical sequences retrieved from both polar marine areas exist for example for Nitrosospira \{Hollibaugh et al., $2002 \# 375\}$ or for ammonia-oxydizing Chrenarchaeota \{Kalanetra et al., $2009 \# 377\}$. In fact one of the latter sequences was found to be dominant during the dark winter period in the Arctic Ocean \{Alonso-Saez et al., 2012 \#378\}. Another interesting example is that of cyanobacteria isolated from mats in ponds from both Polar zones, Nadeau et al. found that psychrophilic strains had almost identical sequences in their $16 \mathrm{~S}$ rDNA, while psychrotolerant strains were more different.

There were no differences in diversity estimates between poles for the three components of microbial life. Nevertheless, the results based on community structure, phylogenetic composition, and shared OTUs, all point to a consistent biogeographical pattern segregating both poles. The only exception was that the bacterial assemblages were not significantly different in terms of phylogenetic content. This pattern had already been observed for the bacterial assemblages of Antarctic vs. Scandinavian lakes (Logares et al., 2013), as well as for the bacterial and microeukaryotic assemblages present in cryoconite holes from both poles (Cameron et al., 2012).

\section{Relationships among the Three Microbial Components}

We found a significant correlation between bacterial and microeukaryotic community structures. However, such link disappeared when accounting for the overall phylogenetic content of the communities. These results indicate that while the overall phylogenetic compositions of each of these microbial groups are not coordinated, there are particular associations between several of their OTUs. Similar findings have recently been reported for a single marine site (Chow et al., 2014). While these signals may simply reflect the associated OTUs' preferences for similar environmental parameters, they could also indicate true biotic interactions.

Our analyses also showed a significant correlation among bacterial and T4-like virus assemblages present in the cellular fractions, although in this case only when accounting for the phylogenetic relatedness among OTUs. Links between viral and bacterial community compositions had previously been detected in a large scale study of sub-polar and Arctic marine sites (Winter et al., 2013). The results from the latter study, however, were not conclusive, since observed links were regionally restricted and failed to show such pattern for the entire dataset. The circumstance that we did not observe such pattern using the free virus dataset may relate to increased noise, since this community may be dominated by viral strains recently arising from exponential outbursts. Nevertheless, the fact that such pattern was detected when accounting for phylogenetic relatedness among OTUs is in line with the idea that many viral strains may infect more likely phylogenetically-related hosts, although this predominant idea has not yet been properly addressed (Flores et al., 2011).

\section{CONCLUSION}

This study represents, to our knowledge, the first massive parallel sequencing-based assessment of three components of microbial life; bacteria, microeukaryotes, and T4-like viruses. In this regard, we believe this study offers an important complement to recent efforts to monitor these relationships following time-series analysis of a single marine ecosystem employing Automated Ribosomal Intergenic Spacer Analysis (ARISA) and terminal restriction fragment length polymorphism (T-RFLP) of phylogenetic marker genes (Needham et al., 2013; Chow et al., 2014). These reports were able to show particular temporal dynamics among some members of the studied communities. Here we have assessed the role of biogeographical and limnological patterns in driving community composition, as well as global co-variation among the different components of microbial life. Overall, the results derived from this study support previous reports on the composition of polar freshwater microbial communities. They are also in agreement with environmental filtering representing a predominant factor driving community structure, with geographical distance effects appearing only at large scales. Also, the noticeable fraction of shared OTUs among sites seemingly indicates reduced dispersal limitations for microorganisms in these ecosystems (Logares et al., 2013). The availability of deep-sequencing phylogenetic marker genes data has allowed the study of community structure no only based on OTUs' relative abundance, but also on overall phylogenetic content. The instances when these two complementary approaches have disagreed have provided important information regarding the ecology of the ecosystems. For example, compositional shifts along the narrow limnological space sampled at the Antarctic sites were only detected based on OTUs' relative abundances, but not when taking into account their phylogenetic relationships. Since ecological coherence and gene conservation have been shown to be negatively correlated with phylogenetic distance (Philippot et al., 2010; Zaneveld et al., 2010), a plausible explanation is that small differences in limnological parameters shifted OTU-level community structure, yet the overall link between ecological function and phylogenetic structure remained stable.

\section{AUTHOR CONTRIBUTIONS}

AA, DA, and DP conceived the study. DA undertook all wet-lab procedures. DA, CP analyzed the data and wrote the manuscript, with input from the other contributors.

\section{ACKNOWLEDGMENTS}

This work was funded by the Spanish Polar Programme and the Spanish Ministry of Science and Innovation grants CTM2008-05134-E/ANT, CTM2009-08644-E, and 
CTM2011-15091-E/ANT. We thank Roche for their support to the Antarctic expedition. DA was supported by the Marie Curie IIF grant PIIF-GA-2012-328287. We thank the logistic support from the Maritime Technology Unit (CSIC) and Las Palmas crew (Spanish Navy). We thank the Mapping and Geographic Information Centre and D. Hodgson (British Antarctic Survey, UK) for their help in identifying suitable water bodies along the Antarctic Peninsula. We thank A. López-Bueno, J. M. Alonso-Lobo, M. L. Avila, L. Little and the students of

\section{REFERENCES}

Aguirre de Cárcer, D., Denman, S. E., McSweeney, C., and Morrison, M. (2011a). Evaluation of subsampling-based normalization strategies for tagged high-throughput sequencing data sets from gut microbiomes. Appl. Environ. Microbiol. 77, 8795-8798. doi: 10.1128/aem.05491-11

Aguirre de Cárcer, D., Denman, S. E., McSweeney, C., and Morrison, M. (2011b). Strategy for modular tagged high-throughput amplicon sequencing. Appl. Environ. Microbiol. 77, 6310-6312. doi: 10.1128/aem.05146-11

Aguirre de Carcer, D., Lopez-Bueno, A., Pearce, D. A., and Alcami, A. (2015). Biodiversity and distribution of polar freshwater DNA viruses. Sci. Adv. 1:e1400127. doi: 10.1126/sciadv.1400127

Alonso-Saez, L., Waller, A. S., Mende, D. R., Bakker, K., Farnelid, H. Yager, P. L., et al. (2012). Role for urea in nitrification by polar marine Archaea. Proc. Natl. Acad. Sci. U.S.A. 109, 17989-17994. doi: 10.1073/pnas.1201914109

Azam, F., Fenchel, T., Field, J., Gray, J., Meyer-Reil, L., and Thingstad, F. (1983). The ecological role of water-column microbes in the sea. Mar. Ecol. Progr. Ser. Oldendorf 10, 257-263.

Bano, N., and Hollibaugh, J. T. (2002). Phylogenetic composition of bacterioplankton assemblages from the Arctic Ocean. Appl. Environ. Microbiol. 68, 505-518. doi: 10.1128/AEM.68.2.505-518.2002

Barberán, A., and Casamayor, E. O. (2014). A phylogenetic perspective on species diversity, beta-diversity and biogeography for the microbial world. Mol. Ecol. 23, 5868-5876. doi: 10.1111/mec.12971

Bates, S. T., Clemente, J. C., Flores, G. E., Walters, W. A., Parfrey, L. W., Knight, R., et al. (2013). Global biogeography of highly diverse protistan communities in soil. ISME J. 7, 652-659. doi: 10.1038/ismej.2012.147

Baty, F., Facompré, M., Wiegand, J., Schwager, J., and Brutsche, M. (2006). Analysis with respect to instrumental variables for the exploration of microarray data structures. BMC Bioinformat. 7:422. doi: 10.1186/1471-2105-7-422

Bragg, L., Stone, G., Imelfort, M., Hugenholtz, P., and Tyson, G. W. (2012). Fast, accurate error-correction of amplicon pyrosequences using Acacia. Nat Meth 9, 425-426. doi: 10.1038/nmeth. 1990

Brum, J. R., and Sullivan, M. B. (2015). Rising to the challenge: accelerated pace of discovery transforms marine virology. Nat. Rev. Micro. 13, 147-159. doi: 10.1038/nrmicro3404

Cameron, K. A., Hodson, A. J., and Osborn, A. M. (2012). Structure and diversity of bacterial, eukaryotic and archaeal communities in glacial cryoconite holes from the Arctic and the Antarctic. FEMS Microbiol. Ecol. 82, 254-267. doi: 10.1111/j.1574-6941.2011.01277.x

Caporaso, J. G., Kuczynski, J., Stombaugh, J., Bittinger, K., Bushman, F. D., Costello, E. K., et al. (2010). QIIME allows analysis of highthroughput community sequencing data. Nat. Methods 7, 335-336. doi: 10.1038/nmeth.f.303

Chow, C. E. T., Kim, D. Y., Sachdeva, R., Caron, D. A., and Fuhrman, J. A. (2014). Top-down controls on bacterial community structure: microbial network analysis of bacteria, T4-like viruses and protists. ISME J. 8, 816-829. doi: 10.1038/ismej.2013.199

Clokie, M. R., Millard, A. D., and Mann, N. H. (2010). T4 genes in the marine ecosystem: studies of the T4-like cyanophages and their role in marine ecology. Virol. J. 7, 7-291. doi: 10.1186/1743-422X-7-291

Comeau, A. M., and Krisch, H. M. (2008). The capsid of the T4 phage superfamily: the evolution, diversity, and structure of some of the most prevalent proteins in the biosphere. Mol. Biol. Evol. 25, 1321-1332. doi: 10.1093/molbev/msn080 the University Center in Svalbard Master Course AB-327 on Arctic Microbiology (2011-2012) for their help collecting and processing the freshwater samples.

\section{SUPPLEMENTARY MATERIAL}

The Supplementary Material for this article can be found online at: http://journal.frontiersin.org/article/10.3389/fmicb. 2016.00337
Convey, P., and Stevens, M. I. (2007). Antarctic biodiversity. Science 317, 1877-1878. doi: 10.1126/science.1147261

Davison, A. C., and Hinkley, D. V. (1997). Bootstrap Methods and Their Applications. Cambridge: Cambridge University Press.

DeSantis, T. Z., Hugenholtz, P., Larsen, N., Rojas, M., Brodie, E. L., Keller, K., et al. (2006). Greengenes, a chimera-checked 16S rRNA gene database and workbench compatible with ARB. Appl. Environ. Microbiol. 72, 5069-5072. doi: 10.1128/AEM.03006-05

Dray, S., Pavoine, S., and Aguirre de Cárcer, D. (2015). Considering external information to improve the phylogenetic comparison of microbial communities: a new approach based on constrained Double Principal Coordinates Analysis (cDPCoA). Mol. Ecol. Resour. 15, 242-249. doi: 10.1111/1755-0998.12300

Fancello, L., Trape, S., Robert, C., Boyer, M., Popgeorgiev, N., Raoult, D., et al. (2013). Viruses in the desert: a metagenomic survey of viral communities in four perennial ponds of the Mauritanian Sahara. ISME J. 7, 359-369. doi: 10.1038/ismej.2012.101

Filée, J., Tétart, F., Suttle, C. A., and Krisch, H. M. (2005). Marine T4-type bacteriophages, a ubiquitous component of the dark matter of the biosphere. Proc. Natl. Acad. Sci. USA. 102, 12471-12476. doi: 10.1073/pnas.0503404102

Flores, C. O., Meyer, J. R., Valverde, S., Farr, L., and Weitz, J. S. (2011). Statistical structure of host-phage interactions. Proc. Natl. Acad. Sci. 108, E288-E297. doi: 10.1073/pnas.1101595108

Ghiglione, J. F., Galand, P. E., Pommier, T., Pedrós-Alió, C., Maas, E. W., Bakker, K., et al. (2012). Pole-to-pole biogeography of surface and deep marine bacterial communities. Proc. Natl. Acad. Sci. U.S.A. 109, 17633-17638. doi: 10.1073/pnas.1208160109

Hollibaugh, J. T., Bano, N., and Ducklow, H. W. (2002). Widespread distribution in polar oceans of a $16 \mathrm{~S}$ rRNA gene sequence with affinity to Nitrosospiralike ammonia-oxidizing bacteria. Appl. Environ. Microbiol. 68, 1478-1484. doi: 10.1128/AEM.68.3.1478-1484.2002

Izaguirre, I., Vinocur, A., Mataloni, G., and Pose, M. (1998). Phytoplankton communities in relation to trophic status in lakes from Hope Bay (Antarctic Peninsula). Hydrobiologia 369-370, 73-87. doi: 10.1023/a:1017070415024

Jones, A. C., Liao, T. S., Najar, F. Z., Roe, B. A., Hambright, K. D., and Caron, D. A. (2013). Seasonality and disturbance: annual pattern and response of the bacterial and microbial eukaryotic assemblages in a freshwater ecosystem. Environ. Microbiol. 15, 2557-2572. doi: 10.1111/1462-2920.12151

Kalanetra, K. M., Bano, N., and Hollibaugh, J. T. (2009). Ammonia-oxidizing Archaea in the Arctic Ocean and Antarctic coastal waters. Environ. Microbiol. 11, 2434-2445.

Lindström, E. S., Kamst-Van Agterveld, M. P., and Zwart, G. (2005). Distribution of typical freshwater bacterial groups is associated with $\mathrm{pH}$, temperature, and lake water retention time. Appl. Environ. Microbiol. 71, 8201-8206. doi: 10.1128/aem.71.12.8201-8206.2005

Logares, R., Lindström, E. S., Langenheder, S., Logue, J. B., Paterson, H., LaybournParry, J., et al. (2013). Biogeography of bacterial communities exposed to progressive long-term environmental change. ISME J. 7, 937-948. doi: 10.1038/ismej.2012.168

López-Bueno, A., Tamames, J., Velazquez, D., Moya, A., Quesada, A., and Alcami, A. (2009). High diversity of the viral community from an Antarctic lake. Science 326, 858-861. doi: 10.1126/science.1179287

Martiny, J. B., Eisen, J. A., Penn, K., Allison, S. D., and Horner-Devine, M. C. (2011). Drivers of bacterial beta-diversity depend on spatial scale. 
Proc. Natl. Acad. Sci. U.S.A. 108, 7850-7854. doi: 10.1073/pnas.10163 08108

McCliment, E. A., Nelson, C. E., Carlson, C. A., Alldredge, A. L., Witting, J., and Amaral-Zettler, L. A. (2012). An all-taxon microbial inventory of the Moorea coral reef ecosystem. ISME J. 6, 309-319. doi: 10.1038/ismej. 2011.108

Needham, D. M., Chow, C. E., Cram, J. A., Sachdeva, R., Parada, A., and Fuhrman, J. A. (2013). Short-term observations of marine bacterial and viral communities: patterns, connections and resilience. ISME J. 7, 1274-1285. doi: 10.1038/ismej.2013.19

Newton, R. J., Jones, S. E., Eiler, A., McMahon, K. D., and Bertilsson, S. (2011). A guide to the natural history of freshwater lake bacteria. Microbiol. Mol. Biol. Rev. 75, 14-49. doi: 10.1128/MMBR.00028-10

Philippot, L., Andersson, S. G., Battin, T. J., Prosser, J. I., Schimel, J. P., Whitman, W. B., et al. (2010). The ecological coherence of high bacterial taxonomic ranks. Nat. Rev. Microbiol. 8, 523-529. doi: 10.1038/nrmicro2367

Quast, C., Pruesse, E., Yilmaz, P., Gerken, J., Schweer, T., Yarza, P., et al. (2013). The SILVA ribosomal RNA gene database project: improved data processing and web-based tools. Nucleic Acids Res. 41, D590-D596. doi: 10.1093/nar/ gks1219

R Core Team (2015). R: A Language and Environment for Statistical Computing. Vienna: R Foundation for Statistical Computing. Available online at: http:// www.R-project.org/

Sul, W. J., Cole, J. R., Jesus Eda, C., Wang, Q., Farris, R. J., Fish, J. A., et al. (2011). Bacterial community comparisons by taxonomy-supervised analysis independent of sequence alignment and clustering. Proc. Natl. Acad. Sci. U.S.A. 108, 14637-14642. doi: 10.1073/pnas.1111435108

Vick-Majors, T. J., Priscu, J. C., and Amaral-Zettler, L. A. (2014). Modular community structure suggests metabolic plasticity during the transition to polar night in ice-covered Antarctic lakes. ISME J. 8, 778-789. doi: 10.1038/ismej.2013.190

Vincent, W. F. (2000). Evolutionary origins of Antarctic microbiota: invasion, selection and endemism. Antarct. Sci. 12, 374-385. doi: $10.1017 /$ S0954102000000420
Wharton, R., Parker, B., and Simmons, G. (1983). Distribution, species composition and morphology of algal mats in Antarctic dry valley lakes. Phycologia 22, 355-365. doi: 10.2216/i0031-8884-22-4-355.1

Wichels, A., Biel, S. S., Gelderblom, H. R., Brinkhoff, T., Muyzer, G., and Schütt, C. (1998). Bacteriophage diversity in the North Sea. Appl. Environ. Microbiol. 64, 4128-4133.

Wilkins, D., Yau, S., Williams, T. J., Allen, M. A., Brown, M. V., DeMaere, M. Z., et al. (2013). Key microbial drivers in Antarctic aquatic environments. FEMS Microbiol. Rev. 37, 303-335. doi: 10.1111/1574-6976.12007

Williamson, S. J., Rusch, D. B., Yooseph, S., Halpern, A. L., Heidelberg, K. B., Glass, J. I., et al. (2008). The Sorcerer II Global Ocean Sampling Expedition: metagenomic characterization of viruses within aquatic microbial samples. PLoS ONE 3:0001456. doi: 10.1371/journal.pone.0001456

Winter, C., Matthews, B., and Suttle, C. A. (2013). Effects of environmental variation and spatial distance on Bacteria, Archaea and viruses in sub-polar and arctic waters. ISME J. 7, 1507-1518. doi: 10.1038/ismej.2013.56

Yannarell, A. C., and Triplett, E. W. (2005). Geographic and environmental sources of variation in lake bacterial community composition. Appl. Environ. Microbiol. 71, 227-239. doi: 10.1128/aem.71.1.227-239.2005

Zaneveld, J. R., Lozupone, C., Gordon, J. I., and Knight, R. (2010). Ribosomal RNA diversity predicts genome diversity in gut bacteria and their relatives. Nucleic Acids Res. 38, 3869-3879. doi: 10.1093/nar/gkq066

Conflict of Interest Statement: The authors declare that the research was conducted in the absence of any commercial or financial relationships that could be construed as a potential conflict of interest.

Copyright (c) 2016 Aguirre de Cárcer, Pedrós-Alió, Pearce and Alcamí. This is an open-access article distributed under the terms of the Creative Commons Attribution License (CC BY). The use, distribution or reproduction in other forums is permitted, provided the original author(s) or licensor are credited and that the original publication in this journal is cited, in accordance with accepted academic practice. No use, distribution or reproduction is permitted which does not comply with these terms. 